\title{
Tratamiento del quilotórax postoperatorio por toracoscopia tras la administración oral de una dieta rica en grasas
}

\author{
Víctor Valentía, David Martínez-Cecilia ${ }^{b}$, Ángeles Gilc y Alberto Martínez-Isla ${ }^{d}$ \\ aDepartamento de Cirugía General. Clínica Universitaria de Navarra. Pamplona. Navarra. España.

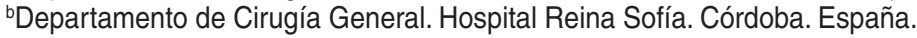 \\ 'Departamento de Cirugía General. Hospital Ramón y Cajal. Madrid. España. \\ dWest London Upper Gastrointstinal Cancer Center. Londres. Reino Unido.
}

El quilotórax postoperatorio tras una esofagectomía es una complicación poco frecuente pero que puede ocasionar graves alteraciones hidroelectrolíticas, hipoproteinemia, deficiencia inmunitaria y problemas respiratorios por compresión pulmonar ${ }^{1}$. La incidencia descrita es de entre el 1 y el $9 \%^{2}$, y puede producirse incluso tras la ligadura profiláctica del conducto torácico, probablemente por las variaciones anatómicas que presenta. Sin tratamiento, la mortalidad puede ascender hasta el $50 \%{ }^{3}$, por eso son importantes el diagnóstico temprano y el correcto tratamiento.

Nuestra experiencia incluye a 2 pacientes varones diagnosticados de adenocarcinoma de esófago $\left(\mathrm{T}_{3} \mathrm{~N}_{1} \mathrm{M}_{0}\right.$, $\mathrm{T}_{4} \mathrm{~N}_{1} \mathrm{M}_{0}$ ) que presentaron un quilotórax tras esofagectomía transhiatal. En el postoperatorio de ambos casos se observó de inmediato la salida, por el drenaje torácico, de un contenido de aspecto turbio, > 1.000-1.500 ml/día, indicio de una fuga del conducto torácico. El diagnóstico se confirmó tras los análisis de laboratorio y la administración de nata concentrada por vía oral (Double Cream, Gaasland Dairy, Bead Foods Pty Ltd, Reino Unido).

Tras manejo médico inicial con restricción de grasas y nutrición parenteral con el que no se logró que el débito se redujera, se decidió la ligadura toracoscópica del conducto torácico. Dos horas antes de la cirugía, se administró en los dos casos $250 \mathrm{~g}$ de nata concentrada por vía oral para hacer patente el conducto torácico.

Los enfermos se colocaron en decúbito lateral izquierdo y se utilizaron tres trocares de 5 y $12 \mathrm{~mm}$ en los espacios intercostales sexto, séptimo y noveno, línea medioaxilar. La administración de la nata concentrada permitió hacer más visible el conducto torácico y localizar la fuga fácilmente al observar salida abundante de contenido le-

Correspondencia: Dr. V. Valentí Azcárate.

Clínica Universitaria de Navarra. Universidad de Navarra.

Avda. Pío XII, 6. 31080 Pamplona. Navarra. España.

Correo electrónico: vvalenti@ unav.es

Manuscrito recibido el 30-8-2007 y aceptado el 28-11-2007. choso. En ambos casos ésta se encontraba distal al cruce con la vena ácigos, por lo que se procedió a ligar el conducto en un punto distal al lugar de la fuga (fig. 1). En el postoperatorio se observó inmediatamente la disminución del contenido lechoso por el drenaje, cuya administración se suspendió a las $48-72$ h para reintroducir la dieta con normalidad y dar el alta a ambos pacientes sin incidencias.

El diagnóstico del quilotórax se debe sospechar cuando hay un incremento importante en el débito del drenaje o una colección pleural recurrente, que se puede confirmar mediante la administración oral o por sonda nasogástrica de compuestos ricos en grasas. Esto constituye la prueba clínica más fiable, puesto que se observa, inmediatamente después de la toma, un aumento en el débito del drenaje, con cambio en la coloración y el aspecto debido al transporte de las grasas absorbidas por el sistema linfáti$\mathrm{CO}^{4}$. En el Reino Unido se utiliza clásicamente la nata con-

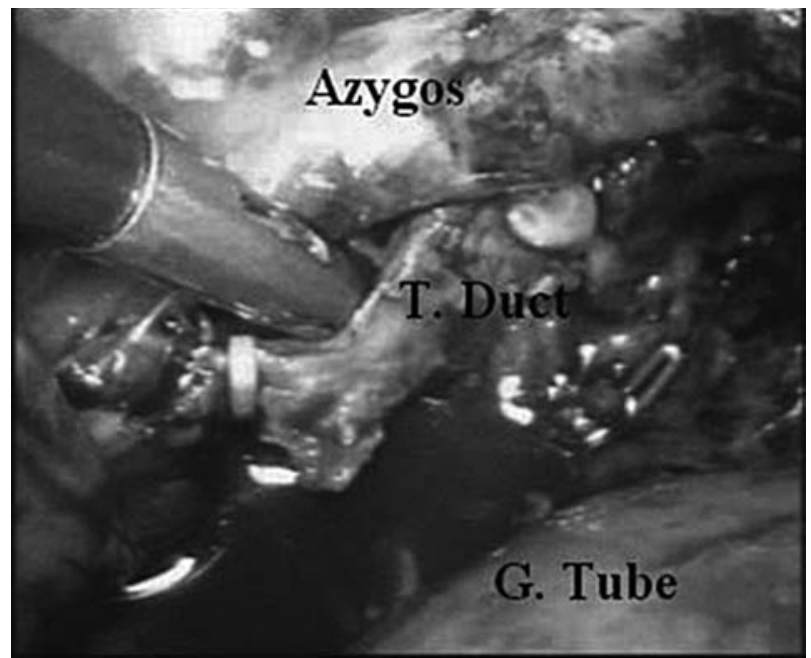

Fig. 1. Toracoscopia en la que se identifica el conducto torácico seccionado y ligado en relación con las distintas estructuras anatómicas. 
centrada que contiene un $47,5 \%$ de grasas totales cada $100 \mathrm{~g}$ en comparación con la crema sencilla, que contiene entre el 10 y el $12 \%$ de grasas. En España, en los casos en que se administra algún preparado, suelen ser mezclas de huevo con leche y aceite. Asimismo, el análisis de una toma del fluido del drenaje muestra un contenido en triglicéridos y linfocitos superior a los valores en plasma.

El tratamiento adecuado es controvertido, y se han publicado buenos resultados con tratamiento conservador en pacientes con buen estado general y drenaje torácico $<2$ l/día ${ }^{2}$. Los principios del tratamiento conservador son reducir el flujo de quilo, drenar la cavidad pleural, prevenir las complicaciones sépticas, aportar nutrición enteral con triglicéridos de cadena media que van directamente al sistema portal o nutrición parenteral. La reducción del flujo quiloso podría favorecerse también con somatostatina o etilefrina ${ }^{5}$. La radiología intervencionista también puede ser útil en el tratamiento de esta complicación con la embolizacion percutánea del conducto torácico, bien sea por medio de coils, bien con sustancias adhesivas ${ }^{6}$. Hay autores que mantienen durante 12 días el tratamiento médico y, si en ese tiempo el débito del drenaje es > $500 \mathrm{ml}$, deciden la reintervención ${ }^{7}$. En cualquier caso parece que el límite para el tratamiento conservador se cifra en un máximo de 2 semanas.

La lesión del conducto torácico raramente es reconocible en el quirófano porque normalmente se encuentra colapsado por el ayuno y presenta cierta dificultad para ser identificado en el campo operatorio, por lo que algunos cirujanos lo ligan por profilaxis, con lo que se reduce significativamente la mortalidad por quilotórax después de una esofagectomía ${ }^{8}$. Los lugares preferidos son el abdomen superior y el tórax inferior, donde la anatomía es más constante. En caso de no identificarlo, una ligadura masiva del tejido entre la aorta y la vena ácigos puede ser eficaz. La administración de nata concentrada previa a la intervención facilita enormemente la localización del conducto, puesto que lo ingurgita, y en caso de producirse una lesión accidental se observa inmediatamente salida de contenido blanquecino lechoso en el punto lesionado.
La ligadura del conducto torácico también ha sido realizada por medio de la cirugía mínimamente invasiva, en la actualidad es una técnica segura y efectiva que minimiza el trauma de la toracotomía porque conlleva menos dolor postoperatorio y morbilidad ${ }^{9,10}$. Con el objetivo de reducir la estancia hospitalaria, consideramos que la reintervención temprana debe ser realizada en pacientes con un importante débito por el drenaje sin esperar a una resolución más tardía con tratamiento médico, puesto que, gracias a las técnicas mínimamente invasivas y a la administración de la nata concentrada, esta intervención se puede realizar de manera sorprendentemente sencilla, rápida y con mínimo trauma al enfermo.

\section{Bibliografía}

1. Fahimi H, Casselman FP, Mariani MA, Van Bowen WJ, Knaepen PJ, Van Swieten HA. Current management of postoperative chylotorax. Ann Thorac Surg. 2001;71:448-50.

2. González JJ, Sanz-Álvarez L, Marqués-Álvarez L, Navarrete-Guijosa F, Martínez-Rodríguez E. Complicaciones de la cirugía de exéresis del cáncer de esófago. Cir Esp. 2006;80:349-60.

3. Schumacher G, Weidemann H, Langrehr JM, et al. Transabdominal ligation of the thoracic duct as a treatment of choice for postoperative chylothorax after esophagectomy. Dis Esophagus. 2007;20:1923.

4. Orriger MB, Bluett M, Deeb GM. Aggressive treatment of chylothorax complicating transhiatal oesophagectomy for carcinoma. Surgery. 1998;104:720-6.

5. Guillen P, Billeret V, Houcke ML, Triboulet JP. Successful management of post-esophagectomy chylothorax/chyloperitoneum by etilefrine. Dis Esophagus. 1999;12:155-6.

6. Cope C. Management of chylotorax via percutaneous embolization. Curr Opin Pulm Med. 2004;10:311-4.

7. Dugue L, Sauvanet A, Farges O, Goharin A, Le Mee J, Belghiti J. Output of chyle as an indicator of treatment for chylothorax complicating oesophagectomy. Br J Surg. 1998;85:1147-9.

8. Bolger C, Walsh TN, Tanner WA, Hennessy TP. Chylothorax after oesophagectomy. Br J Surg. 1991;78:587-8.

9. Bonavina L, Saino G, Bona D, Abraham M, Peracchia A. Thoracoscopic management of chylothorax complicating esophagectomy. J Laparoendosc Adv Surg Tech A. 2001;11:367-9.

10. Kent RP, Pinson RW. Thoracoscopic ligation of the thoracic duct. Surg Endosc. 1993;7:52-3 